\title{
Recent trends in predictive biomarkers for determining malignant potential of oral potentially malignant disorders
}

\author{
Gargi S. Sarode, ${ }^{1}$ Sachin C. Sarode, ${ }^{1}$ Nikunj Maniyar, ${ }^{1}$ Nilesh Sharma, ${ }^{2}$ Sujata Yerwadekar, ${ }^{3}$ \\ Shankargouda Patil ${ }^{4}$
}

${ }^{1}$ Department of Oral Pathology and Microbiology, Dr. D. Y. Patil Dental College and Hospital, Dr. D. Y. Patil

Vidyapeeth, Pune, Maharashtra, India; ${ }^{2}$ Cancer and Translational Research Lab, Dr. D. Y. Patil Biotechnology and

Bioinformatics Institute, Dr. D.Y. Patil Vidyapeeth, Pune, Maharashtra, India; ${ }^{3}$ Department of Orthodontics and

Dentofacial Orthopedics, Dr. D. Y. Patil Dental College and Hospital, Dr. D.Y. Patil Vidyapeeth, Pune, Maharashtra, India; ${ }^{4}$ Department of Maxillofacial Surgery and Diagnostic Sciences, Division of Oral Pathology, College of Dentistry, Jazan University, Jazan, Kingdom of Saudi Arabia

\begin{abstract}
Despite of the tremendous advancements in the field of cancer prevention, detection and treatment, the overall prognosis of oral squamous cell carcinoma (OSCC) still remains poor. This can be partly imparted to the lack of early detection of oral potentially malignant disorders (OPMDs), especially those at a higher risk of progression into OSCC. Over years, various specific and non-specific markers have been introduced that could predict the malignant transformation of OPMDs; however detail information on these OPMD markers in a concise manner is lacking. Moreover, their use on daily clinical basis still remains questionable. With continuous research in the field of cytology and genomics, several contemporary biomarkers have been discovered that are not yet foregrounded and proved to be more promising than those used conventionally. Here, in the present paper, we overview several recently concluded predictive biomarkers with special emphasis on their role in molecular pathogenesis of OSCC transformation. These markers can be used for risk assessment of malignant transformation in patients with OPMDs as well as for prophylactic conciliation and fair management of the high-risk OPMD patient group.
\end{abstract}

Correspondence: Sachin C. Sarode, Department of Oral Pathology and Microbiology, Dr. D. Y. Patil Dental College and Hospital, Dr. D.Y. Patil Vidyapeeth, Sant-Tukaramnagar, Pimpri, Pune - 18, Maharashtra, India. Tel.: +91.9922491465.

E-mail: drsachinsarode@gmail.com

Key words: Biomarkers; molecular pathogenesis; oral potentially malignant disorder; oral cancer; oral squamous cell carcinoma.

Received for publication: 20 March 2019.

Revision received: 7 July 2019

Accepted for publication: 5 August 2019.

This work is licensed under a Creative Commons Attribution NonCommercial 4.0 License (CC BY-NC 4.0).

CC Copyright: the Author(s), 2019

Licensee PAGEPress, Italy

Oncology Reviews 2019; 13:424

doi:10.4081/oncol.2019.424

\section{Introduction}

Head and neck squamous cell carcinoma (HNSCC) which include oral squamous cell carcinoma (OSCC), is ranked as the 6th most commonly encountered cancer in the world; with around 600,000 new cases detected annually. ${ }^{1} 94 \%$ of oral cavity and oropharyngeal cancer cases worldwide are found to be OSCC. ${ }^{2}$ The advancements in the field of cancer detection and treatment has benefited most malignancies yet fails to yield significant prognosis with OSCC, as most of the patients are diagnosed at an advanced disease stages. 3,4 Virtually almost all the cases of OSCCs are preceded by clinically conspicuous but variable changes in the oral mucosa that are collectively referred to as oral potentially malignant disorders (OPMDs). ${ }^{5}$ Effectively addressing such premalignant disorders at an early stage facilitates to arrest progression into OSCC. The rate of malignant transformation of such OPMDs into OSCC fluctuates based on multiple factors like population, gender, habits and the dysplasia grade. Amongst the established OPMDs, early diagnosis of high risk OPMDs is of utmost priority to truncate both morbidity and mortality rates. ${ }^{6}$ Traditionally, grade of oral epithelial dysplasia is the most commonly used indicator to determine the risk of malignant transformation of OPMDs. However, this histologic method is meager and may involve subjectivity resulting in inaccurate results. ${ }^{7}$ Moreover, studies have shown a significant number of lesions that lack dysplastic changes microscopically before progression into OSCC ${ }^{8,9}$ Hence, novel biomarkers that can identify OPMDsthat is at a higher risk for transformation may bestow opportunities for effective pre-emptive intercession in the patient groups at highrisk. With time, several genes and/or proteins are identified as potential markers that would proffer information about the risk of malignant transformation of an OPMD in an individual and also harmonize with the extremity of disease. Biomarkers being products of malignant cells, they may also serve as a target for intervention of therapy to prevent disease progression. ${ }^{10}$ Hence, the need to find biomarkers is essential not only to detect the lesion but also to arrest its transformation into malignancies at its earliest. Even though past advancements in technology has bestowed us with few markers to detect malignant potential of OPMDs, continuous research in this field has led to identification of many contemporary biomarkers that are not yet foregrounded. This article attempts to comprehensively review such recently discernedbiomarkers with a special emphasis on their role in molecular pathogenesis of OSCC (Table 1). For better understanding, the biomarkers have been classified into proteins, glycoproteins and nonproteins. 


\section{Protein biomarkers}

\section{ATP-Binding Cassette, G2 Subfamily (ABCG2)}

ATP-Binding Cassette, G2 Subfamily (ABCG2), belongs to the ATP-binding cassette transporter protein family, which has been studied as a molecular basis that maintains the side population phenotype in stem cells. ${ }^{11}$ Successful attempts have been made to isolate such phenotype from various primary tumors and cancer cell lines that include oral cavity, esophagus, nasopharynx and lung cancers. ${ }^{12}$ Owing to its propensity to eliminate Hoechst 33342 dye, ABCG2 is regarded as a molecular element that perpetuates the side population phenotype in stem cells. ${ }^{13}$ Recent studies have shown that the side population cells own properties similar to cancer stem cells and they play a significant role in oral carcinogenesis. ${ }^{14}$ As a stem cell marker, ABCG2 is overexpressed in a subset of cancer stem-like cells from OSCC. ${ }^{15}$ About $43 \%$ of patients with oral leukoplakias, $21 \%$ with oral lichen planus and $67 \%$ with OSCC exhibited ABCG2 expression. Such expression patterns may evince a step-by-step transformation of an OPMD to malignancy. In a study by Liu et al. ${ }^{16}$ the expression of ABCG2 in OPMDs was reported to be associated with 4.09 fold $(\mathrm{P}=0.01)$ increased risk of malignant transformation. As reported by Shi et al. ${ }^{17}$ the co-expression of Podoplanin and ABCG2 in oral lichen planus was crucially linked with a risk of OSCC transformation. All these results conclude that $\mathrm{ABCG} 2$ may act as a potential biomarker to predict malignant transformation of OPMDs.

\section{Aldehyde dehydrogenase-1}

ALDH1, an isoform of aldehyde dehydrogenase, called cytosolic detoxifying isoenzyme in human cells. It oxidizes aldehydes within the cell and also accords to the oxidation of retinol into retinoic acid in stem cell differentiation at an early stage. ${ }^{18}$ (Figure 1) Based on the reports of various studies, ALDH1 has been delineated as a tumor marker that identifies HNSCC stem cells. It plays a pivotal bit part in the maintenance of the selfregenerating properties and tumorigenicity of cancer stem cells in HNSCC including OSCC. ${ }^{19}$ Visus and co-workers ${ }^{20}$ have perceived over-expression of ALDH1 in oral tissue samples with dysplastic changes and in HNSCC samples ( $\mathrm{P}=0.037)$. ALDH1 serves to distinguish malignant cells from pre-malignant cells thus suggesting its potential to be used as a biomarker for evaluating the risk of malignant transformation of potentially malignant disor$\operatorname{ders}^{21}$ (Figure 1).

Table 1. Various biomarkers for predicting malignant potential of OPMDs and their specificity with role in oral carcinogenesis.

\begin{tabular}{|c|c|c|c|c|c|}
\hline Sr. no. & Name to the marker & Abbreviation & Phenotype & OPMD specificity & Role in carcinogenesis \\
\hline \multicolumn{6}{|c|}{ Protein biomarkers } \\
\hline 1. & $\begin{array}{l}\text { ATP-Binding Cassette, } \\
\text { G2 Subfamily } 16\end{array}$ & ABCG2 & Protein over-expression & Oral leukoplakia & Maintains cancer cell phenotype \\
\hline 2. & Aldehyde dehydrogenase $1^{21}$ & ALDH1 & Protein expression & Oral leukoplakia & $\begin{array}{l}\text { Maintains self-renewal properties } \\
\text { and tumorigenecity of cancer stem cells }\end{array}$ \\
\hline 3. & $\begin{array}{l}\text { B lymphoma Mo-MLV } \\
\text { insertion region } 1 \\
\text { homolog }^{16}\end{array}$ & BMI-1 & Protein over-expression & Oral leukoplakia & Self-renewal of cancer stem cells \\
\hline 4. & E-cadherin $^{34}$ & - & Protein under-expression & Oral leukoplakia & $\begin{array}{l}\text { Controls cell motility, migration } \\
\text { of dysplastic cells and cell invasion }\end{array}$ \\
\hline 5. & $\begin{array}{l}\text { Fibroblast growth factor-2 } \\
\text { and Fibroblast growth factor } \\
\text { receptor-2 }{ }^{40}\end{array}$ & $\begin{array}{l}\text { FGF2 and } \\
\text { FGFR2 }\end{array}$ & Protein over-expression & Not specified & Angiogenesis and cancer cell invasion \\
\hline 6. & $\begin{array}{l}\text { Human EAG-related } \\
\text { gene }^{52}\end{array}$ & HERGl & Protein over-expression & Oral leukoplakia & $\begin{array}{l}\text { Tumor cell proliferation and } \\
\text { differentiation }\end{array}$ \\
\hline 7. & $\begin{array}{l}\text { Minichromosome } \\
\text { maintenance protein }^{56}\end{array}$ & MCM & Protein over-expression & Oral leukoplakia & $\begin{array}{l}\text { Associated with rate of cancer } \\
\text { cell differentiation }\end{array}$ \\
\hline 8. & Phospholipase C- $\gamma 1^{66}$ & PLC 1 & Protein expression & Not specified & Cancer cell migration and invasion \\
\hline 9. & Survivin73 & - & Protein expression & Not specified & Cell proliferation and angiogenesis \\
\hline \multicolumn{6}{|c|}{ Glycoprotein markers } \\
\hline 1. & $\mathrm{CD} 133^{77}$ & - & Protein expression & Oral leukoplakia & As a cancer stem-cell marker \\
\hline 2. & Podoplanin ${ }^{21}$ & PDPN & Protein over-expression & Oral leukoplakia & $\begin{array}{l}\text { Acts as a mediator of cancer cell } \\
\text { invasion and metastasis }\end{array}$ \\
\hline 3. & $\begin{array}{l}\text { Small integrin-binding } \\
\text { ligand N-linked } \\
\text { glycoproteins }^{85}\end{array}$ & SIBLINGs & Protein over-expression & Not specified & $\begin{array}{l}\text { Promotes cancer cell proliferation } \\
\text { and metastasis }\end{array}$ \\
\hline 4. & $\begin{array}{l}\text { Squamous cell carcinoma } \\
\text { antigen }^{90}\end{array}$ & SCCA & Protein expression & Not specified & $\begin{array}{l}\text { Renders resistance against apoptosis } \\
\text { to cancer cells }\end{array}$ \\
\hline
\end{tabular}

miRNAs
Not specified
Cancer cell proliferation, differentiation, migration and metastasis 


\section{B lymphoma Mo-MLV insertion region 1 homolog}

BMI-1 belongs to a polycomb group transcription repressor and is known as an oncoprotein. Through regulation of chromatin structure, BMI-1 arbitrates gene silencing. It has been contemplated as a stem cell-associated gene and plays a role in self-renovation of cancer stem cells ${ }^{22}$ (Figure 2). BMI-1 has been verified to be related in tumorigenecity in HNSCC. ${ }^{23,24}$ Over-expression of BMI-1 in a petite sequence of patients with oral dysplasia was reported by Kang and associates. ${ }^{25}$ Liu et al. ${ }^{16}$ demonstrated overexpression of BMI-1 in about 33\% of patients with oral leukoplakia. Expression of BMI-1 was observed to be coupled with a 3.81 fold increased risk of malignant transformation $(\mathrm{P}=0.02){ }^{16}$ Hence, BMI-1 can be speculated to aid in the determination of OPMDs that are at an increased risk for malignant transformation into OSCC (Figure 2).

\section{E-cadherin}

E-cadherin is a cell membrane-associated protein that entails intercellular adhesion. An intercellular adhesion molecule, E-cadherin organizes and perpetuatescell to cell connections. Studies have shown that certain cell signaling pathways that dominates proliferation, differentiation and also apoptosis of cells are synchronized by E-cadherin. ${ }^{26,27}$ Beta-catenin controls the Wnt-signaling pathway and acts as a transcriptional activator implicated in tumor progression. ${ }^{28}$ Also, loss of differentiation and accretion of an invasive phenotype corresponds to E-cadherin mediated dysfunction of cellular adhesion. ${ }^{29,30}$ In epitheial carcinomas like OSCC, cell-cell adhesion that is mediated by E-cadherin is lost with collateral succession towards malignancy. ${ }^{31}$ Transformation of premalignancy into epithelial carcinomas, in many cases is marked by loss of epithelial phenotype and decreased differentiation, during which the epithelial to mesenchymal transition (EMT) and studies have shown that this EMT may help to anticipate OSCC progression and prognosis. The features of EMT such as loss of inter-cellular adhesion, epithelial cell polarity and increased motility can be appreciated in oral dysplasia and in their string to cancer. Since E-cadherin functions to control cell motility, the migration of dysplastic cells and further cell invasion can be corresponded to downregulation of E-cadherin. ${ }^{32,33}$ von Zeidler et $a l .{ }^{34}$ in one of his studies has shown an inverse co-relation between expression of E-cadherin and epithelial dysplasia grades i.e. a decrease in expression of E-cadherin with higher histopathological dysplasia grade $(\mathrm{P}=0.019)$. Further, down expression of E-cadherin

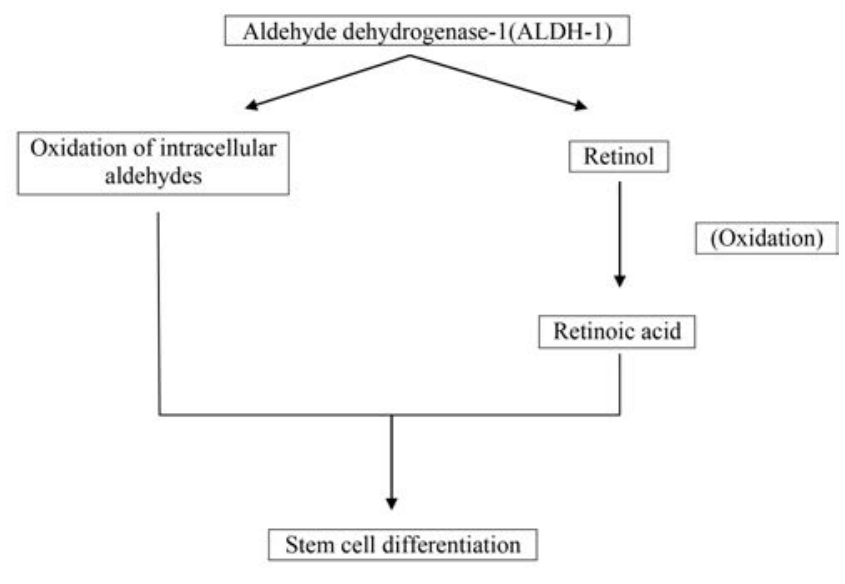

Figure 1. Role of ALDH1 leads to stem cell differentiation. can lead to phenotypic changes in initial stages of oral carcinogenesis as demonstrated by Freitas Silva et al., ${ }^{28}$ thus highlighting Ecadherin down regulation as the central event in EMT. Hence it can be inferred that as the epithelial cells undergo dysplastic changes, increasing the risk of OSCC transformation, there is a reduction in the expression of E-cadherin proposing its use as a new biomarker to identify OPMDs that are at a higher risk for OSCC transformation (Figure 3).

B lymphoma Mo-MLV insertion region 1 homolog (BMI-1)

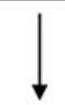

Regulation of chromatin structure

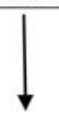

Gene silencing

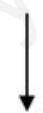

Maintenance of properties of cancer stem cells like self-renewal

Figure 2. Role of BMI-1 in tumor growth.

Dysregulation of E-cadherin

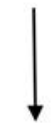

Loss of epithelial phenotype

$\downarrow$

Decreased cellular differentiation

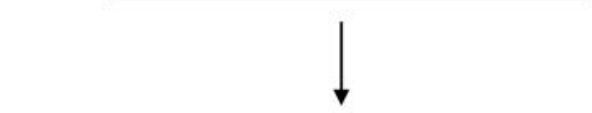

Features of EMT:

- Decreased intercellular adhesion

- Loss of epithelial cell polarity

- Increased cellular motility

Progression of an OPMDtoOSCC 


\section{FGF2 and its receptor FGFR2}

Fibroblast growth factors (FGFs) are associated with cellular growth and differentiation of a wide range of tissue including epithelial cells and also transmit signals among the epithelium and connective tissue. ${ }^{35} \mathrm{FGF}$, a prototype of a huge family of growth factors, truss to low affinity heparin which in turn interacts with high affinity receptors to conciliate cellular response. ${ }^{36,37}$ FGF2 instigates development of new blood vessels and its receptors FGFR2 are associated with production of collagen. ${ }^{38}$ In carcinogenesis, it bit parts a central role in angiogenesis and cancer cell invasion, apart fromfibroblastic proliferationjust about cancer cells in an autocrine or paracrine fashion. ${ }^{39}$ Expression of FGF2 and its receptors FGFR2 has been reported in the neoplastic progression of normal epithelium through dysplasia to OSCC. ${ }^{40}$ FGF2 may be allied in cell mitosis reported in dysplasia, carcinoma in situ and OSCC, as suggested by Janot et al. ${ }^{41}$ and Wakulich et al. ${ }^{42}$ through their separate works that demonstrated well differentiated tumor cells with increased FGF2 staining. Moreover, Wakulichet al. ${ }^{42}$ also reported an augment in FGF2 staining intensity of dysplastic cells through all levels of epithelial dysplasia and carcinoma in situ. Nayak and co-workers ${ }^{40}$ in one of their study found a significant association between FGF2 and FGFR2 expression and malignant transformation of OPMDsto cancers, equally at phenotypic (FGF2 $\mathrm{P}<0.000$, FGFR2 $\mathrm{P}<0.001$ ) and molecular level (FGF2 $\mathrm{P}<0.019$, FGFR2 $\mathrm{P}<0.025$ ). Constitutive signaling of FGFR2 as a result of overexpression of FGFR2 can escort to fortification from apoptosis, a hallmark of cancer. A strong expression of FGF2 in OSCCs than normal tissue, as reported by Myokenet al., ${ }^{43}$ insinuate its association in malignant conversion and self explosion of cancer cells. Hence, immunoexpression of FGF2 and FGFR2 may facilitate us to evaluate progression and malignant transformation of OPMDs (Figure 4).

\section{HERG1}

Modern day research has unveiled expression of ion channels, conduction of ions and electric properties in cancer cells that are inconsistent from the normal cells. ${ }^{44}$ Roughly, about 70 different potassium channels have been recognized out of which the ethera-go-go (EAG) potassium channel family includes three subfamilies: EAG (Kv10); human EAG-related gene (HERG1; Kv11) and ELK (EAG-like; Kv12). ${ }^{45,46}$ Potassium channels are hypothesized to be associated with cellular proliferation and tumor growth. ${ }^{47}$ HERG1 was first discerned in neuroblastoma cells and is formed of 4 subunits, each composed of six transmembrane segments that are organized forming a tetramer surrounding a central aqueous pore. ${ }^{48,49}$ Initially discovered to be accountable for variation in resting potential, HERG1 channel has been involved in the inception and continuation of tumor growth. ${ }^{50}$ Off late it was noted that HERG1 channel is overexpressed in many tumor cell lines and has a role in proliferation and transformation of cancer cells. ${ }^{51}$ In addition to this, HERG1 is also believed to mark an early step for cancer growth and deploy a dictatorial role in cancer cell proliferation and invasion. ${ }^{52}$ In a small study by Fernandez-Valle et al. ${ }^{52}$ HERG1 immunostaining was negative in normal epithelium, whereas it was positive in $36 \%$ of oral leukoplakias $(\mathrm{P}=0.37)$ and in $47 \%$ of OSCC. Though the study fails to mark any statistically significant co-relation between HERG1 protein expression and the risk of OSCC development, the results suggest that HERG1 expression precedes the histopathological signs of OSCC and its expression increases as tumorigenesis advances. ${ }^{52}$

\section{Minichromosome maintenance proteins}

Minichromosome maintenance (MCM) protein family is a small subset of proteins that is recently investigated and found to be closely associated with genomic replication in eukaryotic cells. Constituted of six members from MCM2 to MCM7, the MCM family complex is imperative to DNA replication. These proteins are believed to be crucial components of the typical replication system that safeguards single DNA replication during cell cycle. ${ }^{53-}$ ${ }^{55} \mathrm{MCM}$ family proteins are allied to the pace of cellular differentiation and their expression declines quickly during differentiation and cellular inactivity. Hence, these proteins could be utilized for immunostaining of those cells that holds the potential to enter into the cell cycle. ${ }^{56}$ In a study by Torres-Rendon et al. ${ }^{57}$ expression of MCM2 was notably elevated in such dysplastic lesions that later advanced to cancer. Lameira AG and associates ${ }^{56}$ compared $^{2}$ MCM3 expression amongst the different grades of dysplasia and concluded a statistically significant higher expression of MCM3 in severe dysplasia over mild and moderate groups of dysplasia $(\mathrm{P}<0.01)$. MCM protein antibodies detect more cells that undergo proliferation when compared to some other proliferation markers such as Ki-67 and PCNA thus suggesting it to be a superiorindicator than Ki-67. ${ }^{58}$ Moreover, it was concluded by Endl et al. ${ }^{59}$ that MCM3 is even expressed in cells that have quit proliferation in contrast to $\mathrm{Ki}-67$ that stops expressing in cells with arrested growth. All these reports brings us to conclude that MCM may serve to be a reliable biomarker to predict high risk OPMDs and future research regarding its potentiality should be verified on large patient samples (Figure 5).

\section{Phospholipase C- $\gamma 1$}

Epidermal growth factor receptor (EGFR) performs a noteworthy part in the progress of HNSCC and its expression and atypical gene copy number is allied with an unfavorable prognosis of patients with HNSCC. ${ }^{60,61}$ Recently, Taoudi Benchekroun et al. ${ }^{62}$ demonstrated the relationship of EGFR protein expression and OSCC development in OPMDs that expressed high EGFR.

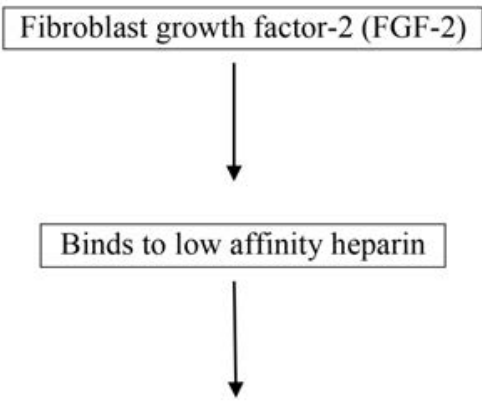

Subsequently interacts with high affinity receptors

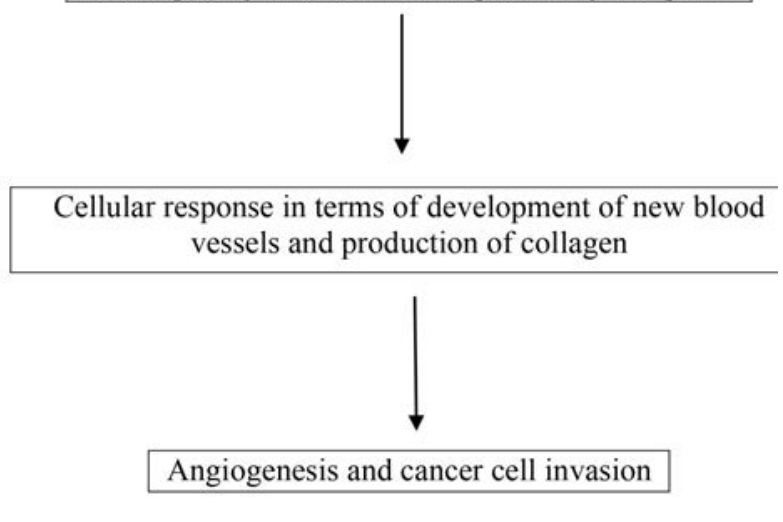

Figure 4. Role of FGF-2 in OSCC transformation. 
Phospholipase C- $\gamma 1$ (PLC $\gamma 1)$ is a crucial molecule in growth factor-reliant signaling transduction and its expression has been reported in invasive cancers that include breast, colorectal, skin and tongue carcinomas. It is shown to be a downstream mark of EGFR signaling. ${ }^{63,64}$ The translocation expression of PLC $\gamma 1$ in OSCC cells is stimulated by EGF, as hypothesized by Xie and coworkers. ${ }^{65}$ Further, the migration of cancer cells during carcinogenesis and invasion of cancer cells also entails PLC $\gamma 1$. Ma LW et al. ${ }^{66}$ conducted a small study to demonstrate correspondence of PLC $\gamma 1$ expression and OSCC development and concluded that those oral potentially malignant lesions which were PLC $\gamma 1$ positive had a appreciablygreaterprevalence of OSCC as compared to those which were PLC $\gamma 1$ negative. Their study showed that cytoplasmic and nuclear PLC $\gamma 1$ positive expression were related with 3.60 times $(\mathrm{P}=0.005)$ and 2.93 times $(\mathrm{P}=0.005)$ higher risk of malignant transformation, respectively. These results of PLC $\gamma 1$ expression in OPMDs favors the significance of PLC $\gamma 1$ in early oral cancer progressions and also suggests its potential to be used as a interpreter of OSCC in high risk OPMDs (Figure 6).

\section{Survivin}

Survivin, a small member of the Inhibitor of Apoptosis (IAP) family of proteins, is expressed in most hematological and solid cancers. ${ }^{67}$ It has a role to play both in cell death regulation and the different aspects of cell division. ${ }^{68}$ It constrains apoptosis through inhibition of capsase activation and surveil proper accomplishment of cell division. Apart from this, Survivin is also implicated in promoting cellular proliferation and angiogenesis. ${ }^{69}$ However, survivin is completely downregulated and untraceable in normal, differentiated mature tissues and is expressed in most of the cancers, including OSCC. Towering expression of survivin in cancer tissues co-relates with more hostile and invasive cancer phenotype. Expression of survivin has also been noted in OPMDs suggesting it to mark an early event during step-wise malignant transformation. ${ }^{70}$ Chen et al. ${ }^{71}$ concluded that the epigenetic and genetic pathways in OSCC were related with IAP expression. Cherukuri and associates $^{72}$ found a significant co-relation between over-expression of survivin and different histological grades of oral dysplasia $(\mathrm{P}<0.05)$. In as study by Lo Muzio et al. ${ }^{73} 94 \%$ of the survivin positive oral pre-cancerous lesions evolved into OSCC. This suggests that survivin, along with other markers, may hold potential to determine increased menace of development from oral dysplasia to carcinoma (Figure 7).

\section{Glycoprotein biomarkers}

\section{CD133}

CD133, also referred as Prominin-1 and AC133, is a pentaspan transmembrane protein. It was originally identified as a hematopoietic stem cell surface marker and as a cell surface marker in early progenitor cells within the human bone marrow. ${ }^{74}$ Advanced studies have lead to conclude the association of CD133 expression with stem cells, tumor cells and phenomenon like regeneration, differentiation and metabolism. It has been identified as a key biomarker for isolation and characterization of lung and head and neck cancer stem cells and is demonstrated to be upregulated in a subset of cancer stem-like cells isolated from OSCC. ${ }^{75,76}$ A cohort study by Liu et al. ${ }^{77}$ reported a positive corelation of immunoexpression of CD133 and poor malignant prognosis of patients with oral leukoplakia. In addition to this, CD133 positive OSCC cancer stem-like cells in OSCC possess exorbitant- ly high cologenicity, invasiveness and tumorigenicity in juxtapose to CD133 negative counterparts as observed by Zhang et al. ${ }^{76}$ The consequential co-relation of CD133 expression in oral leukoplakia $(\mathrm{P}=0.001)$ suggests its potential to be used as a biomarker and predict risk of malignant transformation of oral premalignancies.

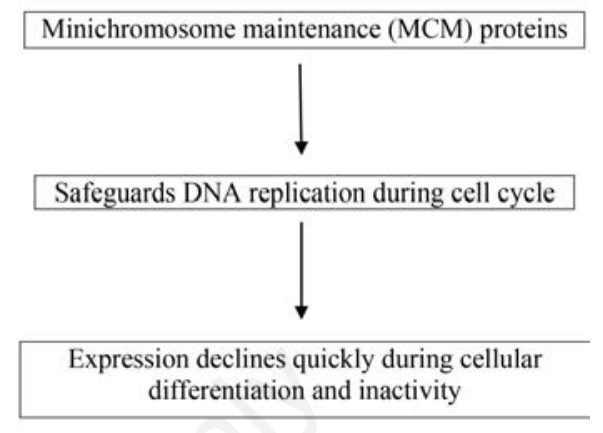

Figure 5. Role of MCM in OSCC transformation.

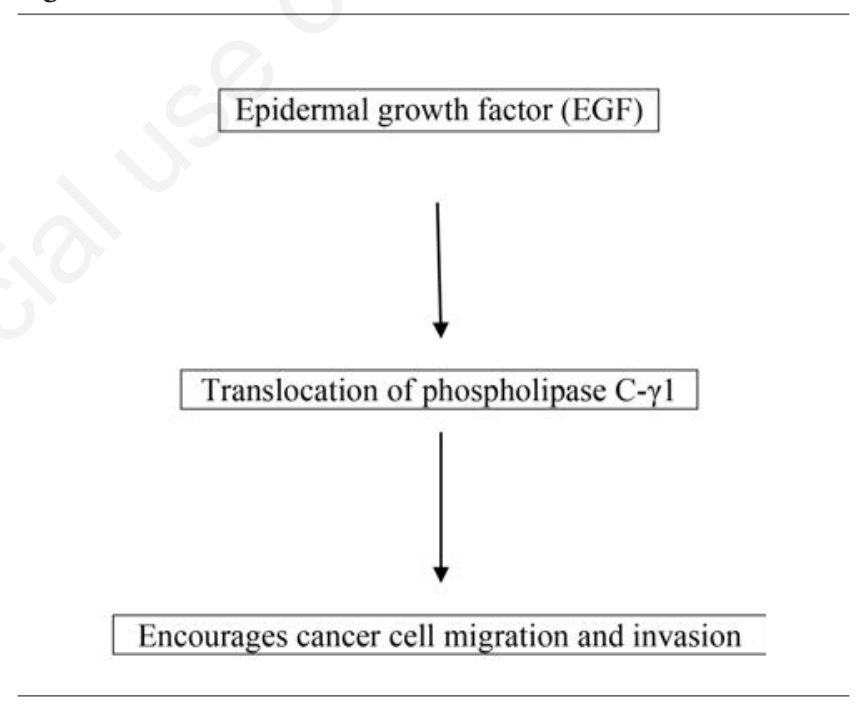

Figure 6. Role of phospholipase C- $\gamma 1$ in OSCC transformation.

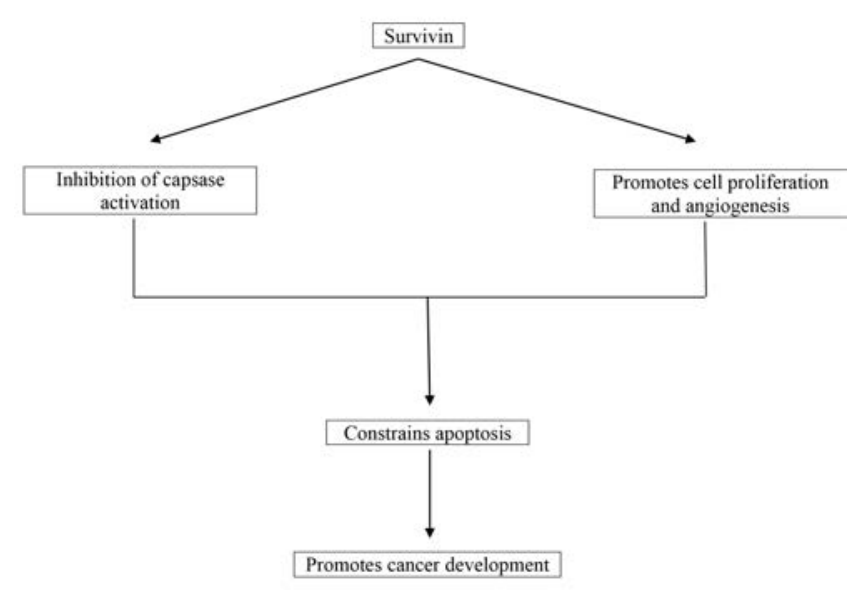

Figure 7. Role of survivin in OSCC transformation. 


\section{Podoplanin}

Podoplanin (PDPN) is a mucin-type of transmembrane glycoprotein, being particularly expressed in lymphatic and not vascular endothelial cells. ${ }^{78}$ It might be active as a arbitrator of invasion and metastasis of cancer cells, as proposed by Wicki and Christofori. ${ }^{79}$ Diverse mechanisms like epithelial-to-mesenchymal transition (EMT), mesenchymal amoeboid transition and collective amoeboid transition that may add to cancer cell migration and dedifferentiation, chiefly relies on dynamic cell cytoskeleton remodeling (Figure 8). Within cancer cells, a greater expression of PDPN is noted in membrane extensions i.e. filopodia and lamellopodia. Perpetuated designs of 3 indispensable residues in cytoplasmic extensions of PDPN bind with ERM proteins and causes improved and continued phosphorylation of ERM proteins. These serve as integral membrane protein and actin cytoskeleton connectors. A decrease in stress fibres and increase in filopodia formation in PDPN positive cell is responsible for EMT. Recent research has shown that CD44, a major hyaluran receptor, is associated with directional persistence of PDPN-positive epithelial cell motility and up-regulation of PDPN and CD44 co-expression was found in aggressive cancer cell lines. ${ }^{80}$ Moreover, tissue samples of oral lichen planus and oral leukoplakia have demonstrated an increased expression of podoplanin. ${ }^{17,81} \mathrm{In}$ addition, podoplanin is also overexpressed in various dysplastic and hyperplastic areas vicinal to the primary OSCC lesion. ${ }^{82}$ In a subsequent study by Habiba et $a l .{ }^{21}$ PDPN overexpression was demonstrated to be strongly associated with malignancy risk $(\mathrm{P}=0.0033)$. All these results suggest that podoplanin overexpression may occur in early oral tumorigenesis and it may serve as a biomarker indicating malignant transformation in premalignancies.

\section{Small integrin-binding ligand N-linked glycoproteins}

Small integrin-binding ligand N-linked glycoproteins (SIBLINGs) comprise of 5 identically oriented integrin-binding glycoproteins: osteopontin (OPN), dentin matrix protein-1 (DMP1 ), bone sialoprotein (BSP), dentin sialophosphoprotein (DSPP), and matrix extra cellular phosphoglycoprotein (MEPE) ${ }^{83}$ By binding to integrins on cell surface and at times with CD44, SIBLINGs act as signal modifiers and fosters cell adhesion, motility and survival in healthy tissues through activation of kinase cascades and transcription factors. Evidences have shown up-regulation of SIBLING family members in several cancers and their involvement in many crucial steps for malignant progression. Cancer cells have the ability to tether to SIBLINGs and their assorted proteolytic wreckage through several integrin recpetors. They promote tumor growth by enhancing cancer cell proliferation and promoting metastasis. ${ }^{84}$ Ogbureke et al. ${ }^{85}$ have reported that while all 5 SIBLINGs are not present in normal oral mucous membrane, BSP, DSPP and OPN are variably over expressed in OSCC. Currently, very little information is available regarding the expression of SIBLINGs in OPMDs. However, in a study by Ogbureke and associates, ${ }^{85}$ expression of DSPP in oral dysplastic lesion was pinpointing of an increase in the likelihood of consequent incidence of local OSCC in comparison to cohort average, autonomous to the degree of epithelial dysplasia ( $\mathrm{P}=0.005)$. In contrast, BSP expression in dysplasia was related with patients that later presented with local OSCC much less often than the cohort average $(\mathrm{P}=0.003)$, with each increase in the level of BSP expression resulting in a decrease in risk $(\mathrm{P}=0.01)$. At present, the mechanism governing the tough statistical connection between SIBLING expression and OSCC transformation potential of OPMDs is not known. There is a possibility that over-expression of DSPP, in spite of BSP expression, is a prerequisite for malignant transformation. Otherwise, the perturbations in the intercellular signaling within dysplastic lesions could by chance amend SIBLING expression exclusive of any direct input to the process of transformation by the SIBLING perse. ${ }^{85}$ Irrespective of the underlying mechanism, it can be thus concluded that the expression of SIBLINGs underscores their potential utility to detect patients at high-risk of OPMD.

\section{Squamous cell carcinoma antigen}

Squamous cell carcinoma antigen (SCCA) along with its 2 isoforms SCCA-1 (SerpinB3) and SCCA-2 (SerpinB4), is a subfraction of the tumor-associated antigen TA-4 and belongs to the clade B subset of serpins. ${ }^{86,87}$ Primarily, SCCA-1 was found to be substantially overexpressed in hepatocellular carcinoma and in squamous cell carcinomas. Later on, added research showed a momentous interchange between overexpression of SCCA-1, more belligerent tumor phenotypes and chemo-resistance in diverse cancers. ${ }^{86,88}$ Through several different mechanisms such as reticence of lysosomal cathepsin proteases, p38 activation or JNK pathway, SCCA-1 grants cancer cells resistance to apoptosis. ${ }^{86}$ Recent studies have verified the role of SCCA-1 in inhibition of generation of reactive oxygen species (ROS) and cell death by means of its impending interface with respiratory Complex I. ${ }^{88}$ Initially isolated from the squamous cell carcinoma of cervix, SCCA reveals to quadrate with the cancer stage, metastasis, treatment outcome and prognosis of the SCC patients, including those with OSCC. ${ }^{89}$ Above normal and even high levels of SCCA has been evident in about one-third of tissue samples from the inactive disease, epithelial dysplasia and active disease group. Such findings propose that cells with dysplasia and OSCC may assemble SCCA that is identi-

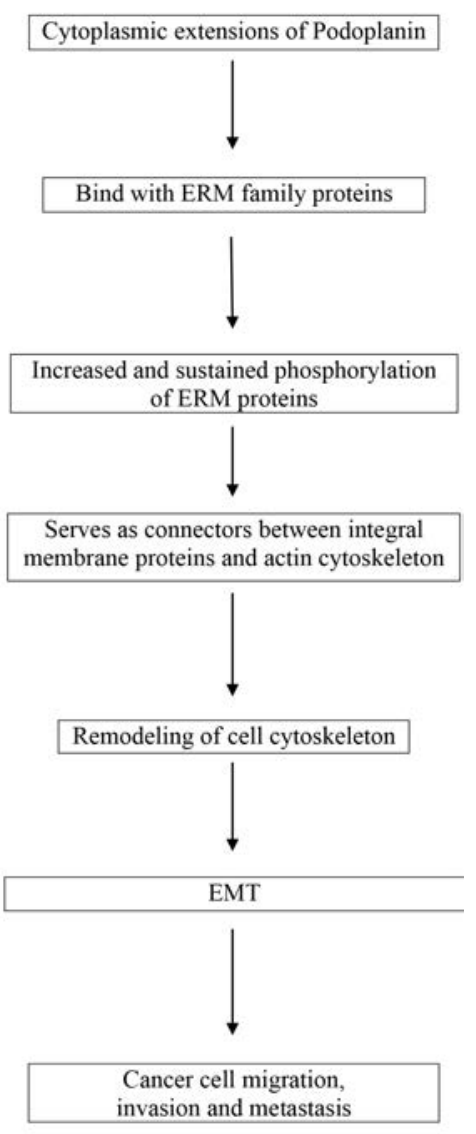

Figure 8. Role of podoplanin in OSCC transformation. 
fiable in patient serum through ELISA $(\mathrm{P}=0.055)$. In a study by Czerninski and co-workers, ${ }^{90}$ a split between above normal levels and the higher $20 \%$ range of normal was observed in patients exhibiting different grades of dysplasia. This suggests a changeover or succession between inactive disease, premalignant disorders and active disease with regard to SCCA production, highlighting the possible contribution of SCCA as a biomarker for detecting malignant potential of OPMDs and monitoring disease progression (Figure 9).

\section{Non-protein biomarker}

\section{miRNAs}

MicroRNAs (miRNAs), a phylogenetically prudent group of dogmatic molecules, are actually tiny endogenous non-protein coding single-stranded RNA molecules. ${ }^{91}$ They modulate target gene expression by binding to protein-coding genes at their messenger RNA sites. ${ }^{92}$ Multiple miRNAs can superintend expression of a single target gene (specific protein) whereas a many target genes can be regulated by a single miRNA (multiple proteins). Mature miRNAs are assimilated into an RNA-induced silencing complex (RISC) through miRNA-miRNA interaction. ${ }^{93}$ This monitors target mRNA (protein) expression by fastening to their 3'untranslated region ( 3 '-UTR) to contribute in many physiological and pathological processes such as cellular growth, multiplication, differentiation, migration, apoptosis, growth control, response to stress, immune response, autophagy, survival, angiogenesis, tumorigenesis, and metastasis. ${ }^{91,94}$ In carcinogenesis, in addition to regulation of expression levels of oncogenes and tumor suppressor genes, miRNAs may also directly purpose as oncogenes or tumor suppressor genes. ${ }^{95}$ Decreased miRNA expression has been revealed to throw in OSCC. ${ }^{96}$ Latest research has shown differential expression of miRNAs amongst OPMD lesions that have distinct histopathological features. ${ }^{97}$ ManymiRNAs have been identified that promises to predict progression of OPMDs to OSCC out of which miRNA-21 and miRNA-31 have been assessed in several studies. Cervigne et al. ${ }^{98}$ noted increased expression of miRNA-21 in oral epithelial cells that showed dysplastic changes $(\mathrm{P}<0.1)$. Xio et al. ${ }^{99}$ assessed expression of miRNA-31 and showed constructive fluorescence in $80 \%$ of cases $(\mathrm{P}<0.5)$ in 5 malignant transformation oral leukoplakias samples out of a total of 15 . However, owing to combinatorial effect a subset of miRNAs could be considered more reliable than just a single microRNA marker. ${ }^{100}$ A panel of overexpressed miRNAs (miRNA-140b, miRNA-181b, miRNA21, miRNA-345, miRNA-518b, miRNA-520g, miRNA-649 and miRNA184) were suggested by Cervigne et al. ${ }^{98}$ to represent premature events in oral carcinogenesis, of which miR-345, miR-21 and miR-181b being directly coupled with amplified severity. miR-196a and miR-196b are expressed during final stages of carcinogenesis and hence identification of circulating miR-196a and miR196b levels may serve as a group for untimely detection of oral cancer. ${ }^{98}$ In a study by Harrandah and co-workers, ${ }^{101}$ lower expression of miR375 and miR-494 and increased expression of miR-21 were corresponded to advancement of OPMDsto oral cancer while an increased expression of miR-375 was identified with non-progressive lesions. miR-375 is credited to be able to differentiate progressive OPMDs from that of non-progressive ones suggesting its expression alone as a potential biomarker to diagnose high risk OPMDs. ${ }^{101}$ Moreover, conducting a retrospective follow-up using 5 years data Philipone and associates ${ }^{102}$ highlighted miRNA-2045p, miRNA-208b-3p, miRNA-129-2-3p and miRNA-3065-5p as markers that can predict progression of oral leukoplakias into carcinomas. Zahram et al. ${ }^{103}$ studied three non-invasive salivary miRNAs (miRNA-21, miRNA-145, miRNA-184) and identified them to be quick analytical biomarkers for predicting oral malignant transformation, of which miRNA184 being the most precise one with AUC of 0.86 . Thus, these current data suggests the use of miRNAs, singly or in combined sets, as risk stratification biomarkers to predict malignant transformation of OPMDs (Figure 10).

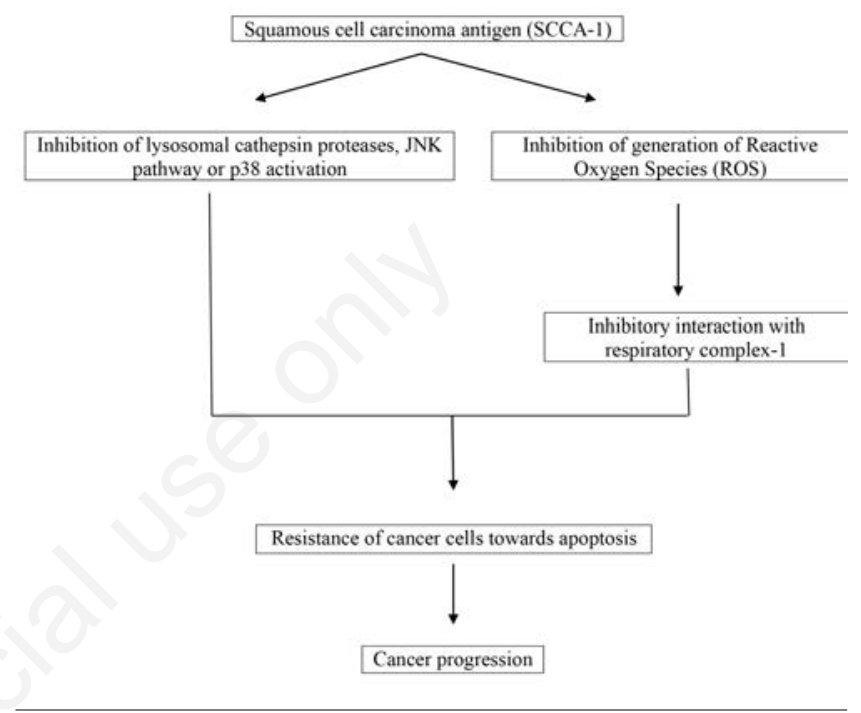

Figure 9. Role of SCCA1 in OSCC transformation.

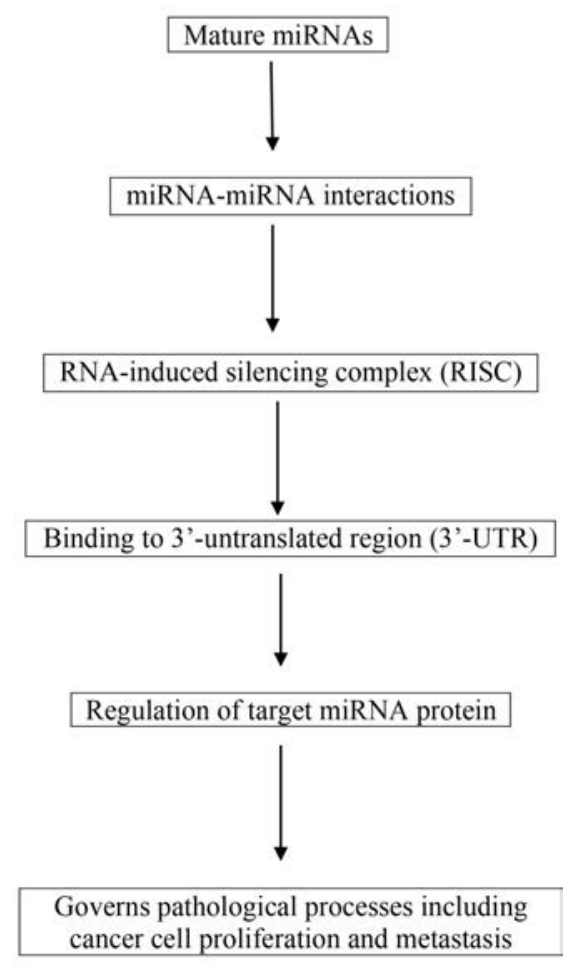

Figure 10. Role of miRNA in OSCC transformation. 


\section{Conclusions}

Though there is no specific well established biomarker for any of the OPMDs as cancer risk assessment tool incorporates not only biomarkers but other variables like demographic and epidemiological, many authors have performed several multivariate analyses to check for the co-expression of more than one biomarker or a cocktail biomarker in OPMDs and co-related the results with risk for malignant transformation. Habiba $\mathrm{U}$ et al. ${ }^{21}$ presented co-expression of ALDH1 and podoplanin as co-factors and found that the risk of malignant transformation was considerably higher for oral leukoplakias with positive co-expression of ALDH1 and podoplanin when compared with oral leukoplakias with negative expression of both the proteins. Similarly, Liu W et al. ${ }^{77}$ studied co-expression of ALDH1 and CD133 and demonstrated a superior prognostic value for their co-expression in oral leukoplakia.

In the present paper, we have tried to comprehensively compile all the potential predictive biomarkers for OPMDs, which can be used for assessing their malignant potential and should be extensively studied further. This is one of its kinds of paper, which describes the role of biomarkers in molecular pathogenesis of OSCC transformation as well. There are many biomarkers, which have been implicated in oral cancer diagnosis like cancer testis antigens, ${ }^{104}$ which should be further exploited in predicting the malignant potential of OPMDs. We also believe that evaluation of various biomarkers should form the basis for classification of the OPMDs, which can be used as the center for treatment planning and predicting the long-term outcome of the patients.

\section{References}

1. Ferlay J, Shin HR, Bray F, et al. Estimates of worldwide burden of cancer in 2008: GLOBOCAN 2008. Int J Cancer 2010;127:2893-917.

2. Mao L, Hong WK, Papadimitrakopoulou VA. Focus on head and neck cancer. Cancer Cell 2004;5:311-6.

3. Jemal A, Clegg LX, Ward E, et al. Annual report to the nation on the status of cancer, 1975-2001, with a special feature regarding survival. Cancer 2004;101:3-27.

4. Haddad RI, Shin DM. Recent advances in head and neck cancer. N Engl J Med 2008;359:1143-54.

5. Sarode SC, Sarode GS, Tupkari JV. Oral potentially malignant disorders: A proposal for terminology and definition with review of literature. J Oral Maxillofac Pathol 2014;18:S77-80.

6. Ganly I, Patel S, Shah J. Early stage squamous cell cancer of the oral tongue-clinicopathologic features affecting outcome. Cancer 2012;118:101-11.

7. Tilakaratne WM, Sherriff M, Morgan PR, et al. Grading oral epithelial dysplasia: analysis of individual features. J Oral Pathol Med 2011;40:533-40.

8. Papadimitrakopoulou VA, Hong WK, Lee JS, et al. Low-dose isotretinoin versus beta-carotene to prevent oral carcinogenesis: long-term follow-up. J Natl Cancer Inst 1997;89:257-8.

9. Silverman S Jr, Gorsky M, Lozada F. Oral leukoplakia and malignant transformation. A follow-up study of 257 patients. Cancer 1984;53:563-8.

10. Carmeliet P, Jain RK. Molecular mechanisms and clinical applications of angiogenesis. Nature 2011;473:298-307.

11. Zhou S, Schuetz JD, Bunting KD, et al. The ABC transporter Bcrp1/ABCG2 is expressed in a wide variety of stem cells and is a molecular determinant of the side-population phenotype. Nat Med 2001;7:1028-34.
12. Zhang P, Zhang Y, Mao L, et al. Side population in oral squamous cell carcinoma possesses tumor stem cell phenotypes. Cancer Lett 2009;277:227-34.

13. Ho MM, Ng AV, Lam S, Hung JY. Side population in human lung cancer cell lines and tumors is enriched with stem-like cancer cells. Cancer Res 2007;67:4827-33.

14. Zen Y, Fujii T, Yoshikawa S, et al. Histological and culture studies with respect to ABCG2 expression support the existence of a cancer cell hierarchy in human hepatocellular carcinoma. Am J Pathol 2007;170:1750-62.

15. Okamoto A, Chikamatsu K, Sakakura K, et al. Expansion and characterization of cancer stem-like cells in squamous cell carcinoma of the head and neck. Oral Oncol 2009;45:633-9.

16. Liu W, Feng JQ, Shen XM, et al. Two Stem Cell Markers, ATP-Binding Cassette, G2 Subfamily (ABCG2) and BMI-1, Predict the Transformation of Oral Leukoplakia to Cancer. Cancer 2012;118:1693-700.

17. Shi P, Liu W, Zhou ZT, et al. Podoplanin and ABCG2: malignant transformation risk markers for oral lichen planus. Cancer Epidemiol Biomarkers Prev 2010;19:844-9.

18. Marcato P, Dean CA, Giacomantonio CA, Lee PW. Aldehyde dehydrogenase: Its role as a cancer stem cell marker comes down to the specific isoform. Cell Cycle 2011;10:1378-84.

19. Chen YC, Chen YW, Hsu HS, et al. Aldehyde dehydrogenase 1 is a putative marker for cancer stem cells in head and neck squamous cancer. Biochem Biophys Res Commun 2009;385:307-13.

20. Visus C, Ito D, Amoscato A, et al. Identification of human aldehyde dehydrogenase 1 family member A1 as a novel CD8+ T-cell-defined tumor antigen in squamous cell carcinoma of the head and neck. Cancer Res 2007;67:10538-45.

21. Habiba U, Hida K, Kitamura T, et al. ALDH1 and podoplanin expression patterns predict the risk of malignant transformation in oral leukoplakias. Oncol Lett 2017;13:321-8.

22. Sparmann A, van Lohuizen M. Polycomb silencers control cell fate, development and cancer. Nat Rev Cancer 2006;6:846-56.

23. Prince ME, Sivanandan R, Kaczorowski A, et al. Identification of a subpopulation of cells with cancer stem cell properties in head and neck squamous cell carcinoma. Proc Natl Acad Sci U S A 2007;104:973-8.

24. Chen YC, Chang CJ, Hsu HS, et al. Inhibition of tumorigenicity and enhancement of radiochemosensitivity in head and neck squamous cell cancer-derived ALDH1-positive cells by knockdown of Bmi-1. Oral Oncol 2010;46:158-65.

25. Kang MK, Kim RH, Kim SJ, et al. Elevated Bmi-1 expression is associated with dysplastic cell transformation during oral carcinogenesis and is required for cancer cell replication and survival. Br J Cancer 2007;96:126-33.

26. Halbleib JM, Nelson WJ. Cadherins in development: cell adhesion, sorting, and tissue morphogenesis. Genes Dev 2006;20:3199-214.

27. Cavallaro U, Christofori G. Cell adhesion and signaling by cadherins and Ig-CAMs in cancer. Nat Rev Cancer 2004;4:118-32.

28. Freitas Silva BS, Yamamoto-Silva FP, Pontes HAR, Pinto Junior DS. E-cadherin downregulation and Twist overexpression since early stages of oral carcinogenesis. J Oral Pathol Med 2014;43:125-31.

29. García AS, Hernández MMA, Sánchez EF, et al. E-cadherin, laminin and collagen IV expression in the evolution from dysplasia to oral squamous cell carcinoma. Med Oral Patol Oral Cir Bucal 2006;11:E100-5.

30. Williams HK, Sanders DSA, Jankowski JAZ, et al. 
Expression of cadherins and catenins in oral epithelial dysplasia and squamous cell carcinoma. J Oral Pathol Med 1998;27:308-17.

31. Hashimoto T, Soeno Y, Maeda G, et al. Progression of oral squamous cell carcinoma accompanied with reduced E-cadherin expression but not cadherin switch. PLoS One 2012;7:e47899.

32. Theveneau E, Mayor R. Cadherins in collective cell migration of mesenchymal cells. Curr Opin Cell Biol 2012;24:677-84.

33. Huber MA, Kraut N, Beug H. Molecular requirements for epithelialmesenchymal transition during tumor progression. Curr Opin Cell Biol 2005;17:548-58.

34. von Zeidler SV, de Souza Botelho T, Mendonça EF, Batista AC. E-cadherin as a potential biomarker of malignant transformation in oral leukoplakia: a retrospective cohort study. BMC Cancer 2014;14:972.

35. Bikfalvi A, Klein S, Pintucci G, Rifkin DB. Biological roles of fibroblast growth factor-2. Endocr Rev 1997;18:26-45.

36. Klagsbrun M, Baird A. A dual receptor system is required for basic fibroblast growth factor activity. Cell 1991;67:229-31.

37. Coutts JC, Gallagher JT. Receptor for fibroblast growth factor. Immunol Cell Biol 1995;73:584-9.

38. Dailey L, Ambrosetti D, Mansukhani A, Basilico C. Mechanisms underlying differential responses to FGF signaling. Cytokine Growth Factor Rev 2005;16:233-47.

39. Brooks AN, Kilgour E, Smith PD. Molecular pathways: fibroblast growth factor signaling: a new therapeutic opportunity in cancer. Clin Cancer Res 2012;18:1855-62.

40. Nayak S, Goel MM, Makker A, et al. Fibroblast Growth Factor (FGF-2) and Its Receptors FGFR-2 and FGFR-3 May Be Putative Biomarkers of Malignant Transformation of Potentially Malignant Oral Lesions into Oral Squamous Cell Carcinoma. PLoS One 2015;10:e0138801.

41. Janot F, el Naggar AK, Morrison RS, et al. Expression of basic fibroblast growth factor in squamous cell carcinoma of the head and neck is associated with degree of histologic differentiation. Int J Cancer 1995;64:117-23.

42. Wakulich C, Jackson-Boeters L, Daley TD, Wysocki GP. Immunohistochemical localization of growth factors fibroblast growth factor- 1 and factors fibroblast growth factor- 2 and factors fibroblast growth factor-3 in normal oral epithelium, epithelial dysplasias, and squamous cell carcinoma. Oral Surg Oral Med Oral Pathol Oral Radiol Endod 2002;9:573-9.

43. Myoken Y, Okamoto T, Sato JD, Takada K. Immunohistochemical localization of fibroblast growth factor-1 (FGF-1) and FGF-2 in oral squamous cell carcinoma (SCC). J Oral Parhol Med 1994;23:451-6.

44. Kunzelmann K. Ion channels and cancer. J Membr Biol 2005;205:159-73.

45. Smith GA, Tsui HW, Newell EW, et al. Functional up-regulation of HERG K1 channels in neoplastic hematopoietic cells. J Biol Chem 2002;277:18528-34.

46. Bauer CK, Schwarz JR. Physiology of EAG K1 channels. J Membr Biol 2001;182:1-15.

47. Wang H, Zhang Y, Cao L, et al. HERG K1 channel, a regulator of tumor cell apoptosis and proliferation. Cancer Res 2002;62:4843-8.

48. Arcangeli A, Bianchi L, Becchetti A, et al. A novel inwardrectifying $\mathrm{K} 1$ current with a cell-cycle dependence governs the resting potential of mammalian neuroblastoma cells. J Physiol 1995;489:455-71.

49. Bianchi L, Wible B, Arcangeli A, et al. Herg encodes a K1 current highly conserved in tumors of different histogenesis: a selective advantage for cancer cells? Cancer Res
1998;58:815-22.

50. Lastraioli E, Taddei A, Messerini L, et al. hERG1 channels in human esophagus: evidence for their aberrant expression in the malignant progression of Barrett's esophagus. J Cell Physiol 2006;209:398-404.

51. Menendez ST, Rodrigo JP, Alvarez-Teijeiro S, et al. Role of HERG1 potassium channel in both malignant transformation and disease progression in head and neck carcinomas. Mod Pathol 2012;25:1069-78.

52. Fernández-Valle Á, Rodrigo JP, Rodríguez-Santamarta T, et al. HERG1 potassium channel expression in potentially malignant disorders of the oral mucosa and prognostic relevance in oral squamous cell carcinoma. Head Neck 2016;38:1672-8.

53. Staed BE, Sorbara CD, Bradl CJ, Davery MJ. ATP binding and hydrolysis by $\mathrm{MCM} 2$ regulate DNA binding by $\mathrm{MCM}$ complexes. J Mol Biol 2009;391:301-13.

54. Yoshida K. Identification of a novel cell-cycle-induced MCM family protein MCM9. Biochem Biophys Res Commun 2005;331:669-74.

55. Chuang $\mathrm{CH}$, Yang D, Bai G, et al. Post-transcriptional homeostasis and regulation of MCM2-7 in mammalian cells. Nucleic Acids Res 2012;40:4914-24.

56. Lameira AG, Pontes FS, Guimarães DM, et al. MCM3 could be a better marker than Ki-67 for evaluation of dysplastic oral lesions: an immunohistochemical study. J Oral Pathol Med 2014;43:427-34.

57. Torres-Rendon A, Roy S, Craig GT, Speight PM. Expression of Mcm2, geminin and Ki67 in normal oral mucosa, oral epithelial dysplasias and their corresponding squamous-cell carcinomas. Br J Cancer 2009;100:1128-34.

58. Scott IS, Odell E, Chatrath $\mathrm{P}$, et al. A minimally invasive immunocytochemical approach to early detection of oral squamous cell carcinoma and dysplasia. $\mathrm{Br} \mathrm{J}$ Cancer 2006;94:1170-5.

59. Endl E, Kausch I, Baack M, et al. The expression of Ki-67, MCM3, and p27 defines distinct subsets of proliferating, resting, and differentiated cells. J Pathol 2001;195:457-62.

60. Szabo' B, Nelhubel GA, Ka' rpa' ti A, et al. Clinical significance of genetic alterations and expression of epidermal growth factor receptor (EGFR) in head and neck squamous cell carcinomas. Oral Oncol 2011;47:487-96.

61. Sheu JJ, Hua CH, Wan L, et al. Functional genomic analysis identified epidermal growth factor receptor activation as the most common genetic event in oral squamous cell carcinoma. Cancer Res 2009;69:2568-76.

62. Taoudi Benchekroun M, Saintigny P, Thomas SM, et al. Epidermal growth factor receptor expression and gene copy number in the risk of oral cancer. Cancer Prev Res 2010;3:800-9

63. Wells A, Grandis JR. Phospholipase C-c1 in tumor progression. Clin Exp Metastasis 2003;20:285-90.

64. Sala G, Dituri F, Raimondi C, et al. Phospholipase Cc1 is required for metastasis development and progression. Cancer Res 2008;68:10187-96.

65. Xie Z, Chen Y, Liao EY, et al. Phospholipase C-gammal is required for the epidermal growth factor receptor-induced squamous cell carcinoma cell mitogenesis. Biochem Biophys Res Commun 2010;397:296-300.

66. Ma LW, Zhou ZT, He QB, Jiang WW. Phospholipase C- $\gamma 1$ expression correlated with cancer progression of potentially malignant oral lesions. J Oral Pathol Med 2013;42:47-52.

67. Ambrosini G, Adida C, Altieri DC. A novel anti-apoptosis gene, survivin, expressed in cancer and lymphoma. Nat Med 
1997;3:917-21.

68. Kim YH, Kim SM, Kim YK, et al. Evaluation of Survivin as a prognostic marker in Oral Squamous Cell Carcinoma. J Oral Pathol Med 2010;39:368-75.

69. Chaiyarit P, Jintakanon D, Klanrit P, et al. Immunohistochemical analyses of survivin and heat shock protein 90 expression in patients with oral lichen planus. J Oral Pathol Med 2009;38:55-62.

70. Jinbu Y, Tsukinoki K, Miyagi N, et al. Expression of survivin in oral squamous cell carcinoma. Oral Med Pathol 2006;11:41-4.

71. Chen YJ, Lin SC, Kao T, et al. Genome-wide profiling of oral squamous cell carcinoma. J Pathol 2004;204:326-32.

72. Gayathri C, Rao GV. Immunohistochemical expression of Survivin in oral leukoplakia and oral squamous cell carcinoma. J NTR Univ Health Sci 2017;6:39-44.

73. Lo Muzio L, Pannone G, Leonardi R, et al. Survivin, a potential early predictor of tumor progression in the oral mucosa. $\mathrm{J}$ Dent Res 2003;82:923-8.

74. Wu Y, Wu PY. CD133 as a marker for cancer stem cells: progresses and concerns. Stem Cells Dev 2009;18:1127-34.

75. Hsu HS, Huang PI, Chang YL, et al. Cucurbitacin I inhibits tumorigenic ability and enhances radiochemosensitivity in nonsmall cell lung cancer-derived CD133-positive cells. Cancer 2011;117:2970-85.

76. Zhang Q, Shi S, Yen Y, et al. A subpopulation of CD133(b) cancer stem-like cells characterized in human oral squamous cell carcinoma confer resistance to chemotherapy. Cancer Lett 2010;289:151-60.

77. Liu W, Wu L, Shen XM, et al. Expression patterns of cancer stem cell markers ALDH1 and CD133 correlate with a high risk of malignant transformation of oral leukoplakias. Int $\mathrm{J}$ Cancer 2013;132:868-74.

78. Kahn HJ, Marks A. A new monoclonal antibody, D2-40, for detection of lymphatic invasion in primary tumors. Lab Invest 2002;82:1255-7.

79. Wicki A, Christofori G. The potential role of podoplanin in tumor invasion. Br J Cancer 2007;96:1-5.

80. Swain N, Kumar SV, Routray S, et al. Podoplanin - a novel marker in oral carcinogenesis. Tumour Biol 2014;35:8407-13.

81. Saintigny P, El-Naggar AK, Papadimitrakopoulou V, et al. DeltaNp63 over expression, alone and in combination with other biomarkers, predicts the development of oral cancer in patients with leukoplakia. Clin Cancer Res 2009;15:6284-91.

82. Kawaguchi H, El-Naggar AK, Papadimitrakopoulou V, et al. Podoplanin: A novel marker for oral cancer risk in patients with oral premalignancy. J Clin Oncol 2008;26:354-60.

83. Fisher LW, Fedarko NS. Six genes expressed in bones and teeth encode the current members of the SIBLING family of proteins. Connect Tissue Res 2003;44:33-44.

84. Ogbureke KU, Nikitakis NG, Warburton G, et al. Up-regulation of SIBLING proteins and correlation with cognate MMP expression in oral cancer. Oral Oncol 2007;43:920-32.

85. Ogbureke KU, Abdelsayed RA, Kushner H, et al. Two members of the SIBLING family of proteins, DSPP and BSP, may predict the transition of oral epithelial dysplasia to oral squamous cell carcinoma. Cancer 2010;116:1709-17.

86. Turato C, Pontisso P. SERPINB3 (serpin peptidase inhibitor, clade B (ovalbumin), member 3). Atlas Genet Cytogenet Oncol Haematol 2015;19:202-9.

87. Schick C, Pemberton PA, Shi GP, et al. Cross-class inhibition of the cysteine proteinases cathepsins $\mathrm{K}, \mathrm{L}$, and $\mathrm{S}$ by the serpin squamous cell carcinoma antigen 1: a kinetic analysis. Biochemistry 1998;37:5258-66.
88. Ciscato F, Sciacovelli M, Villano G, et al. SERPINB3 protects from oxidative damage by chemotherapeutics through inhibition of mitochondrial respiratory complex I. Oncotarget 2014;5:2418-27.

89. Kato H, Torigoe T. Radioimmunoassay for tumor antigen of human cervical squamous cell carcinoma. Cancer 1977;40:1621-8.

90. Czerninski R, Basile JR, Kartin-Gabay T, et al. Cytokines and tumor markers in potentially malignant disorders and oral squamous cell carcinoma: a pilot study. Oral Dis 2014;20:477-81.

91. Gassling V, Hampe J, Acil Y, et al. Disease-associated miRNA-mRNA networks in oral lichen planus. PLoS One 2013;8:e63015.

92. Chen $\mathrm{H}-\mathrm{C}$, Tseng $\mathrm{Y}-\mathrm{K}$, Chi $\mathrm{C}-\mathrm{C}$, et al. Genetic variants in microRNA-146a $(\mathrm{C}>\mathrm{G})$ and microRNA-1269b $(\mathrm{G}>\mathrm{C})$ are associated with the decreased risk of oral premalignant lesions, oral cancer, and pharyngeal cancer. Arch Oral Biol 2016;72:21-32.

93. Chattopadhyay E, Singh R, Ray A, et al. Expression deregulation of mir31 and CXCL12 in two types of oral precancers and cancer: importance in progression of precancer and cancer. Sci Rep 2016;6:32735.

94. Shi W, Yang J, Li S, et al. Potential involvement of miR-375 in the premalignant progression of oral squamous cell carcinoma mediated via transcription factor KLF5. Oncotarget 2015;6:40172-85.

95. Sun L, Liu L, Fu H, et al. Association of decreased expression of serum miR-9 with poor prognosis of oral squamous cell carcinoma patients. Med Sci Monit 2016;22:289.

96. Lin N, Lin Y, Fu X, et al. MicroRNAs as a novel class of diagnostic biomarkers in detection of oral carcinoma: a metaanalysis study. Clin Lab 2016;62:451-61.

97. Hung KF, Liu CJ, Chiu PC, et al. MicroRNA-31 upregulation predicts increased risk of progression of oral potentially malignant disorder. Oral Oncol 2016;53:42-7.

98. Cervigne NK, Reis PP, Machado J, et al. Identification of a microRNA signature associated with progression of leukoplakias to oral carcinoma. Hum Mol Genet 2009;18:4818-29.

99. Xiao W, Bao Z-X, Zhang C-Y, et al. Upregulation of miR-31* is negatively associated with recurrent/newly formed oral leukoplakia. PLoS One 2012;7:e38648.

100. Prasad G, Seers C, Reynolds E, McCullough MJ. A panel of microRNAs can be used to determine oral squamous cell carcinoma. J Oral Pathol Med 2017;46:940-8.

101. Harrandah AM, Fitzpatrick SG, Smith MH, et al. MicroRNA375 as a biomarker for malignant transformation in oral lesions. Oral Surg Oral Med Oral Pathol Oral Rad 2016;122:743-52.

102. Philipone E, Yoon AJ, Wang S, et al. MicroRNAs-208b-3p, 204-5p, $129-2-3 p$ and $3065-5 p$ as predictive markers of oral leukoplakia that progress to cancer. Am J Cancer Res 2016;6:1537-46.

103. Zahran F, Ghalwash D, Shaker O, et al. Salivary microRNAs in oral cancer. Oral Dis 2015;21:739-47.

104. Zamunér FT, Karia BTR, Zanardo de Oliveira C, et al. A Comprehensive Expression Analysis of Cancer Testis Antigens in Head and Neck Squamous Cell Carcinoma Revels MAGEA3/6 as a Marker for Recurrence. Mol Cancer Ther $2015 ; 14 ; 828-34$. 